\title{
Pre-operative planning of multiple probes in three dimensions for liver cryosurgery: comparison of different optimization methods
}

\author{
Amir Jaberzadeh*, Caroline Essert*
}

Pre-operative planning of percutaneous thermal ablations is a difficult but decisive task for a safe and successful intervention. The purpose of our research is to assist surgeons in preparing cryoablations with an automatic pre-operative path planning algorithm able to propose a placement for multiple needles in 3D. The aim is to optimize several surgical constraints while taking into account a precise computation of the frozen area. Using an implementation of the precise estimation of the iceballs, this study focuses on the optimization in an acceptable time of multiple probes positions with 5 degrees of freedom, regarding the constraint of optimal volumetric coverage of the tumor by the combined necrosis. Pennes equation was used to solve the propagation of cold within the tissues, and included in an objective function of the optimization process. The propagation computation being time-consuming, seven optimization algorithms from the literature were experimented under different conditions and compared, in order to reduce overall computation time while preserving precision. Moreover, several hybrid algorithms were tested to reduce required time for the computations. Some of these methods were found suitable for the conditions of our cryosurgery planning. We conclude that this combination of bioheat simulation and optimization can be appropriate for a use by practitioners in acceptable conditions of time and precision.

Keywords: Surgery planning, Derivative free optimization, Bioheat simulation.

\section{Introduction}

Minimally invasive surgery has known an increasing interest in the past decades [1]. The small size of incisions is beneficial to patients by decreasing the discomfort as well as the time required for recovery compared to conventional surgery, all with the same benefits. Percutaneous cryoablation is a good example, in which the cancerous tissue is frozen using one or multiple needles. During this procedure, tissue temperature drops to $-40^{\circ} \mathrm{C}$ around needle tip, which is lethal for cells included in the iceball volume [2]. The final goal of cryotherapy is the necrosis of cancerous cells while preserving surrounding healthy tissue and avoiding damages to vital anatomical structures. For this purpose, an accurate surgical planning needs to be done beforehand by surgeons.

However, the non-invasive, real-time monitoring of three-dimensional isotherm surface of this critical temperature within the tissue during cryosurgical procedures has remained a challenge. Since temperature can be measured only at discrete points in the target region, simulation of heat transfer is an extremely useful tool to estimate the real coverage for a candidate probe placement. A number of models has been proposed to solve the bioheat propagation equation in two and three dimensions[3].

An important parameter in cryosurgery planning is the optimal choice of cryoprobes locations with specific shapes and dimensions. It is typically done in a trial-and-error task to find the best configuration. Since the manufactured cryoprobes have been produced with a limited set of active lengths and diameters, and freezing protocol is commonly fixed, other cryosurgical parameters such as number of cryoprobes and cryoprobe placement are good candidates for optimization and planning during the procedure.

The overall objective of our research is to provide the surgeon with an automatic pre-operative path planning algorithm able to propose a placement for multiple needles in 3D, taking into account several surgical constraints as well as a precise 
computation of the frozen area. In this paper, we focus on the optimization of the tridimensional placement of multiple needles to cover the tumor at best with the estimated $-40^{\circ} \mathrm{C}$ isotherm surface. We first explain the implementation of the accurate simulation of the propagation of cold within the tissues to generate the isotherm surface. This simulation is timeconsuming, and needs to be repeated at each iteration of the optimization process. Therefore in a second part we compare several optimization approaches under different conditions to find the most suitable in terms of compromise between speed and accuracy, in order to be able to propose to the surgeon a good positioning strategy in a reasonable time. Then we complete this study by proposing a hybrid approach with a choice of several possible combinations of optimization functions.

\section{Context}

\subsection{Related works}

The simulation of heat propagation involving freezing and heating of biological tissues was investigated in [3]. In this paper, authors formulated cryosurgery and hyperthermia with a unified equation that can be applied to frozen, partially frozen and unfrozen tissue regions.

Different types of thermal ablations naming Radiofrequency Ablation (RFA) and cryosurgery require an optimal needle positioning. Altrogge[4] proposed an optimization method based on the simulation of temperature within tissues. However the resulting trajectory does not take into account surrounding organs and no computation time is indicated. In [5] authors studied treatment planning of RFA based on a constraint concept for automatically computing insertion trajectories. Socalled hard constraints are used to determine the insertion zones on the skin. The quality of the trajectories that are allowed according to the hard constraints is then rated by so-called soft constraints that represent clinically relevant parameters such as the distance to critical structures.In another paper a method for multiple needle placement of RFA was proposed. Several spheroids were used to cover the tumor and a distance map was computed to determine needles size and location [6]. Moreover, in [7] the authors coupled a finite element method of RFA and a new local optimization method based on image processing algorithms so, the objective function is viewed as the correlation of two scalar volumetric images. The first image describes the relative importance of a point in the target domain and the second image represents the likelihood of achieving complete ablation at a given position relative to the electrode. They worked with 1 to 3 probes but they consider a lot of predefined geometrical conditions for tumor shape and also their timing were not recorded for comparison.

The problem of multiple needle optimization for cryosurgery was first addressed by Keanini and Rubinsky [8] using simplex method. The heat transfer equation was solved for a 3D domain with finite-difference method. Authors optimized only the number of cryoprobes and their geometrical dimensions (diameter and active length), but optimization of other parameters, such as cryoprobe placement in the target tissue and their thermal protocol, seems to be more practical. They used an idealized model and geometry for urethral warmer, prostate, bladder and rectum. In 2001 Baissalov et al. [9] studied simultaneous optimization of cryoprobe placement and thermal simulation using a gradient descent algorithm called L-BFGS$\mathrm{B}$ method. They described a 3D solution based on the cumulative 2D transverse planes, but the shown results were only for $2 \mathrm{D}$ state in a prostate model.

Tanaka and Rubin [10] used a mechanical based method to solve the problem of cryoprobe optimization in two phases. Phase I called bubble-packing starts with generating ellipsoidal elements (or bubbles) inside planning domain, then van der Waals'-like forces are simulated to move these bubbles until a minimum-force configuration is found. A single bioheat simulation is executed at the end of Phase I. The simulation is terminated at the point at which a minimum defect region is found for that particular layout. In phase II, a bioheat equation is solved and a new set of forces on the cryoprobes are computed based on the defect region and one or more cryoprobes are moved accordingly. This survey was done in $2 \mathrm{D}$ for the prostate while in 2008, the same team extended their work to 3D [11] but just for bubble packing method.

Giorgi et al. [12] used Ants Colony (ACO) to choose the optimal parameter configurations. Computation of the cost function is based on the numerical solution of several direct Stefan problems solved by a Euler-Galerkin approach. This method combines a finite difference approximation of the time-derivative and a finite element approach solving the spacedependent part of the differential problem. This study was done on a $2 \mathrm{D}$ standard prostate phantom.

\subsection{Problem statement}

As mentioned above all previous studies were done for prostate cryosurgery in which needles are placed in the same direction and consequently the number of optimization variables is reduced. In this paper the first goal is to deal with a general case in which the planning domain could be $3 \mathrm{D}$ and the needles have 5 degrees of freedom: 3 translations for position and 2 rotations for orientation (the rotation around the own axis of the needle is not considered).

Previous studies have computed bioheat propagation in order to have a more realistic simulation of cryosurgery procedure. Bioheat propagation in the tissue is affected by needle parameters, time and surrounding tissues. An interesting source of bioheat is the flowing blood within large adjacent vessels which can cause a "heat sink" effect and may prevent temperature from decreasing to lethal levels. This may result in inadequate ablation, thus increasing the risk of tumor recurrence in this region [13]. Our second objective is to consider surrounding tissues inside the bioheat equation computation, as they have an important role in forming the final frozen region. 
Our problem of simultaneous optimization of thermal protocol and cryoprobes placement requires handling a large number of bound constrained optimization variables and ability to minimize an objective function that cannot be expressed analytically in terms of optimization variables.

High computational cost of bioheat equation in each iteration requires a fast converging optimization method for real time purposes. Optimization algorithms have been studied in the literature to find the most suitable ones in terms of convergence and computational time while avoiding local minima. We experimented several of them in order to perform comparisons in the conditions of our problem: local optimization methods such as Generating Set Search or Nelder Mead, and global optimization methods such as Genetic Algorithm, Simulated Annealing, Multilevel Coordinate Search, Surrogate Modeling and Evolutionary Strategy were tested. Our final goal is to optimize trajectories of needles quickly and precisely, while taking into account a realistic simulation of the formation of iceballs at their tips.

\section{Materials and Methods}

\subsection{Numerical computation of the bioheat transfer within the tissues}

The estimation of the thermal distribution of the cryosurgical procedure is predicted using multiprobe thermal simulations. The results of the simulations are quantified in terms of the isotherm locations in the anatomy at any given time and the value of the objective function in the optimization scheme. These tools provide a means of assessing the effectiveness of the treatment. Our method consists of a time-dependent model of iceball formation based on bioheat transfer equation around the needle tip, taking into account major vessels surrounding the frozen area that influence the freezing process. Most of the theoretical analysis on heat transfer in living tissue are originated from the Pennes equation [14], which describes the influence of blood flow on the temperature distribution in the tissue in terms of volumetrically distributed heat sinks or sources.

This uniform energy equation [3] for biological tissue which can be applied to frozen, partially frozen and unfrozen tissue regions, can be written as:

$$
\tilde{C} \frac{\partial T(X, t)}{\partial t}=\nabla \cdot \tilde{k} \nabla[T(X, t)]-\tilde{\omega}_{b} C_{b} T(X, t)+\tilde{Q}_{m}+C_{b} \tilde{\omega}_{b} T_{a} \quad X \in \Omega(t)
$$

where $\tilde{C}$ is the effective heat capacity; $\tilde{k}(T)$ is the effective thermal conductivity; $\tilde{Q}_{m}$ is the effective metabolic heat generation; $\tilde{w}_{b}(T)$ is the effective blood perfusion; $T_{a}$ is the arterial temperature; $C_{b}$ is the heat capacity of blood; $X$ contains the Cartesian coordinates $\mathrm{x}, \mathrm{y}$, and $\mathrm{z} ; T(X, t)$ is the temperature of tissue; $\Omega(t)$ denotes the domain at time $\mathrm{t}$. The description and derivation of this coefficients in different states are omitted here for brevity. A finite difference algorithm is applied to solve this complex problem with phase change heat transfer in biological tissues. Applying this formulation to Eq 1 and using the following relation to express the linear term $T(X, t)$ on the right side of Eq 1 ,

$$
T(X, t)=\beta T(X, t+\Delta t)+(1-\beta) T(X, t)
$$

where $\beta$ is a relaxation factor, and $0 \leq \beta \leq 1$, Eq 1 can be discretized as follows:

$$
\begin{aligned}
T(X, t+\Delta t)= & \frac{1-W(1-\beta) \Delta t-m \cdot F_{o}}{1+W \beta \Delta t} T(X, t)+\Sigma_{i=1}^{\frac{m}{2}} \frac{F o}{1+W \beta \Delta t} T\left(X+\Delta x_{i}, t\right) \\
& +\Sigma_{i=1}^{\frac{m}{2}} 1 \frac{F o}{1+W \beta \Delta t} T\left(X-\Delta x_{i}, t\right)+\frac{\left(\tilde{Q}_{m}+\tilde{\omega}_{b} C_{b} T_{a}\right) \Delta t}{1+W \beta \Delta t}
\end{aligned}
$$

where $\Delta t$ is the time increment; $W=\tilde{\omega}_{b} C_{b} / \tilde{C}$, and $F_{O}=\tilde{k} \cdot \Delta t / \tilde{C} \cdot \Delta x^{2}$ is the Fourier number; $m=2,4,6$ correspond to the cases of one, two and three dimensions respectively, and in order to avoid numerical instability, the space and time steps are limited by $1-W(1-\beta) \Delta t-m . F_{o} \geqslant 0$. Applying the boundary conditions at time $t+\Delta t$ and substituting the calculated results at the previous time $\mathrm{t}$, the unknown $\mathrm{T}$ at time $t+\Delta t$ can be solved from the above equation.

The tissue domain is prescribed in a rectangular geometry with $7 \times 7 \times 7 \mathrm{~cm}$ in the $\mathrm{x}, \mathrm{y}$ and $\mathrm{z}$ directions respectively, in which $\mathrm{x}$ denotes the tissue depth from the skin surface while $\mathrm{y}$ and $\mathrm{z}$ are along the surface. The boundary conditions at the probe surface are prescribed respectively according to probe tip and probe shank as: $T=-196^{\circ} \mathrm{C}$ at probe tip; $\partial T / \partial n=0$ at probe shank. The initial temperature in tissue is simplified as $T 0=37^{\circ} \mathrm{C}$. In calculations, the grid resolution is $\Delta x=\Delta y=\Delta z=1 \mathrm{~mm}$ and $\Delta t=0.1 \mathrm{~s} . \tilde{Q}_{m}=0$ in a highly vascularized tissue like liver and $\tilde{w}_{b}=0.005$.

This simulation of thermal propagation is used in our routine described below:

1. The location of liver, tumor, skin and vessels are determined thanks to a segmentation process performed on the images. In this step, a 3D mesh for each anatomical structure are reconstructed. All vessels are considered as fixed sources of heat at $37^{\circ} \mathrm{C}$

2. The shape of needle tip has been designed to fit real cryoprobes

3. Needle tip is placed at an initial position and orientation inside the tumor manually or by the optimization method 
4. The simulation procedure imitates a standard cryoablation pattern that consists of two 10 minutes freezing intervals and one 5 minutes thawing step in between, which is congruent with needle manufacturer calibration data in a tissue-like gel, which from now on we call reference data

5. The iceballs are obtained by a $3 \mathrm{D}$ reconstruction of the $-40^{\circ} \mathrm{C}$ isotherm surfaces. The selected value was chosen according to surgeons needs, as this temperature is used as a threshold to determine the resulting necrosis volume

Steps 4 and 5 are intended to be repeated at each iteration of the optimization process.

\subsection{Optimization of the probes placement in $3 D$}

The optimization process allows to refine the number and placement of the needles (3 translations and 2 rotations for each) to minimize a defect function. It is based on an iterative procedure including the bioheat equation resolution at each iteration (above mentioned steps 4 and 5). Every resolution begins with the assumption that the placement of cryoprobes is given with a fixed tip temperature of $-196^{\circ} \mathrm{C}$ while the initial temperature of tumor and background tissue is $37^{\circ} \mathrm{C}$. Vessels are considered to have an invariant temperature of $37^{\circ} \mathrm{C}$. Then, the bioheat equation can be solved. The resulting temperature field is processed to evaluate the defect function providing a quantitative estimate of the mismatch between the frozen tissue and the target tissue. Eq.4 shows the definition of the cost function.

More formally, a specific configuration of the cryosurgery design is represented by a state variable $U$, which is a list of $N$ operating parameters (position of cryoprobes). Their admissible values are contained in $S \subset R^{N}$. The cost function is the defect weighting function $F: S \rightarrow \mathbb{N}$ such that:

$$
F\left(\theta_{U}\right)=\int_{V} \mu\left(\theta_{U}(x)\right) d x
$$

where $\theta_{U}$ is the temperature distribution associated to $U$ and

$$
\mu(\theta(x))= \begin{cases}0 & \text { if } \theta(x)<\tilde{\theta} \text { and } x \text { is diseased } \\ 1 & \text { if } \theta(x)<\tilde{\theta} \text { and } x \text { is healthy } \\ 1 & \text { if } \theta(x) \geq \tilde{\theta} \text { and } x \text { is diseased } \\ 0 & \text { if } \theta(x) \geq \tilde{\theta} \text { and } x \text { is healthy }\end{cases}
$$
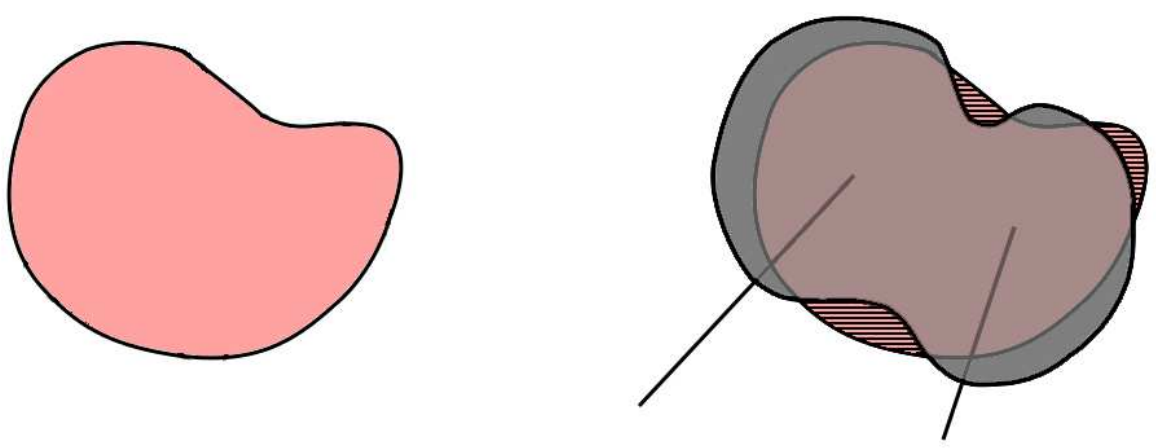

Figure 1. Schematic 2D representation of the defect region. The targeted tumor is represented in pink on the left part of the figure. On the right part, the interacting iceballs are drawn in gray over the tumor. The objective of the optimization is to maximize the amount of frozen tumor (pink-gray) and minimize non damaged tumor parts (striped) and damaged healthy tissue (pure gray).

A schematic view of this function is demonstrated in Fig.1. Optimization algorithms use this objective function and yield the new positions of cryoprobes in order that the next step can begin. The procedure stops when further correction of the position of cryoprobes becomes negligible or the predefined maximum number of iterations is reached.

Conventional optimization techniques typically require multiple evaluations of the cost function for each iteration. For example, gradient based algorithms would require multiple function evaluations to compute the gradients [15]. Keanini and Rubinsky [8] stated that methods which compute explicit derivatives are likely to be inefficient. Our work employs techniques that avoid calculating derivatives so as to minimize the number of simulations.

As mentioned above our optimization algorithm should be capable of handling a large number of bound constrained optimization variables and be able to minimize an objective function that cannot be expressed analytically in terms of the optimization variables. Keeping these parameters in mind, a bound constrained derivative free optimization method which do not stuck in local minima (global optimization) with low number of iterations would be suitable. Our problem is a convex optimization problem because of cubic search domain surrounding the target region. 
Derivative free optimization methods are classified to local and global optimization methods as well as as deterministic, model based and stochastic methods[16]. Seven optimization methods were selected based on the parameters just mentioned in order to compare their strengths and weaknesses to our problem.

- Local methods:

- Deterministic:

Nelder-Mead (NM): This algorithm, also called simplex method, was introduced in [17]. The idea is to create a simplex at each iteration depending on the size of input variables, then try to find the worst corner points of the simplex and substitute them using operations like rejection, expansion and contractions. The simplex converges to a single optimal point.

Generating Set Search (GSS): Generating Set Search method was selected between different Pattern search methods. Each iteration of GSS method consists of two basic steps. The search step is performed first over a finite set of search directions $H_{K}$ generated by some, possibly heuristic, strategy that aims to improve the current iterate but may not guarantee convergence. If the search step fails to produce a better point, GSS method continues with the poll step, which is associated with a generating set that spans positively $R^{\mathrm{n}}$ Generating sets are usually positive bases, with a cardinality between $\mathrm{n}+1$ to $2 \mathrm{n}$ [18].

- Global methods:

- Deterministic:

Multilevel coordinate search (MCS): It partitions the search space into boxes and in each iteration a label is assigned to each box based on the number of times it has been splitted. MCS selects boxes with the lowest objective value for each level value and marks them as candidates for splitting and will converge when the maximum number of predefined levels reached [19].

- Model based:

Surrogate modeling (SM): Building a model of objective functions in our search domain allows us to optimize a function with less number of iterations [20]. In order to build such a model, one should starts with sampling the search domain and construct an initial surrogate model. Then optimizers are used to converge the model, evaluate the best point and update the surrogate model. For this purpose we have employed a mixture of radial basis functions [21] and kriging [22] interpolations for our surrogate model. Radial basis functions approximate given function by considering an interpolating model based on radial functions and then models a deterministic response as a stochastic process by means of a kriging basis function.

- Stochastic:

Simulated annealing (SA): This global optimization algorithm is inspired by the modeling of the physical process of heating a material and then slowly lowering the temperature to decrease defects. It consists in starting from an initial state and bringing the system to another state minimizing the system's energy. This method generates a new trial point $(\hat{x})$ in each iteration which is then compared with the incumbent point $x^{k}$ and will be accepted based on a probability function $P[23]$.

$$
P\left(\hat{x} \mid x_{k}\right)= \begin{cases}\exp \left[\frac{f(\hat{x})-f\left(x_{k}\right)}{T_{k}}\right] & \text { if } f(\hat{x})<f\left(x_{k}\right) \\ 1 & \text { if } f(\hat{x})<f\left(x_{k}\right)\end{cases}
$$

The probability $\mathrm{P}$ depends on the temperature parameter $T_{k}$; the sequence $\left\{T_{k}\right\}$ is referred to as the cooling schedule. Cooling schedules are decreasing sequences that converge to 0 sufficiently slow for the algorithm to scape from a local optima.

Genetic algorithms (GA): This method introduced by Holland [24] resembles natural selection and reproduction processes governed by rules that assure the survival of the fittest in large populations. Individuals (points) are associated with identity genes that define a fitness measure (objective function value). A set of individuals form a population, which adapts and mutates following probabilistic rules that utilize the fitness function.

Evolutionary Strategy (ES): This method belongs to the class of Evolutionary Algorithms called covariance matrix adaptation evolution strategy which uses perturbation with expected zero value and a covariance matrix which is iteratively updated to guide search towards regions with better solutions [25].

\subsection{Experiment design}

Our experiments were done on two patient models from the 3D-IRCADb database. They consist of 3D triangular surface meshes reconstructed from CT images of liver tumors surrounded by vessels and normal liver tissue. To test different conditions, we chose a case with a small tumor and another with a large one.

For the tests we experimented several parameters, each time changing one parameter while others were fixed. Optimization methods, number of needles and size of the tumors were selected as different experimental designs. Seven optimization methods were considered as mentioned in the previous section. In order to compute the defect volume, the bioheat equation was solved in each iteration and the objective function value was computed by comparing the temperature of each point in the tissue domain. 
Comparisons for speed and convergence of the optimization methods were based on the solution profile of each optimizer for few iterations to large ones. We tried to check the sensitivity of each optimizer to other parameters like tumor size and number of electrodes. The tumor size affects the complexity of the problem by extending or shrinking the search domain, while the number of needles modifies the optimization input variables. In order to have a fair comparison, four different experimental designs were selected, two for small tumor and two for large ones. For small tumor we chose to test with 3 and 5 needles, and in the case of large tumor tests were done for 5 and 8 needles in order to have enough necrosis area to cover whole tumor. All solvers had a maximum of 500 iterations.

Algorithm parameters are taken from successful parameter settings in the literature [26] over various problems. We did not try to find the best parameters and unique values for each method and mostly aimed to find a robust method suitable for our problem. The type of croyprobe we modeled was a PERC-24 from Endocare. The theoretical volume of the iceball $\left(-40^{\circ} \mathrm{C}\right.$ isotherm surface) of this cryoprobe type, given by the manufacturer, is $1.6 \times 1.6 \times 3.6 \mathrm{~cm}$. The ratio between theoretical single iceball volume and tumor volume allows to estimate an initial number of needles. We experimented our optimizations on two tumor sizes, small and large. This ratio is 0.7 for small and 0.162 for large tumor, with the chosen cryoprobe type. In order to destroy tumors, it is intuitive to start with a number of needles providing a total iceballs volume at least equal to the tumor volume.

\section{Results and discussion}

\subsection{Simulation results}

In this first subsection we try to highlight the difference between theoretical and simulated iceball shapes and sizes in the vicinity of a vessel. In Fig. 2(b) the blue ellipsoid refers to $0^{\circ} \mathrm{C}$ iceball, the red one is $-20^{\circ} \mathrm{C}$ and the green one is the $-40^{\circ} \mathrm{C}$ isotherm. This simulated shape mimics the manufacturer's standard isotherms stated in the user manual. Then by approaching the needle tip to a vessel (Fig. 2(c)), simulated iceballs start to deform. Fig. 2(a) shows the percentage of volume change compared to its rest position as a function of the distance to the vessel. This deformation can represent more than one forth of the iceball volume. This emphasizes the usefulness of an accurate computation of the deformed shape of the iceball during the optimization process instead of using a simplified ellipsoid, as the result may be misleading for the outcome of the surgery. This difference in shape and volume can affect the optimization results drastically, as it will be shown in the following section.

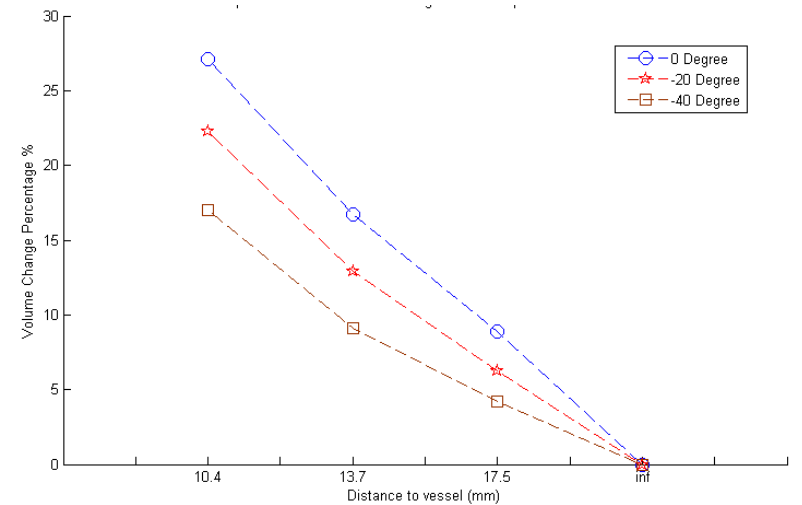

(a) Amount of volume deformation

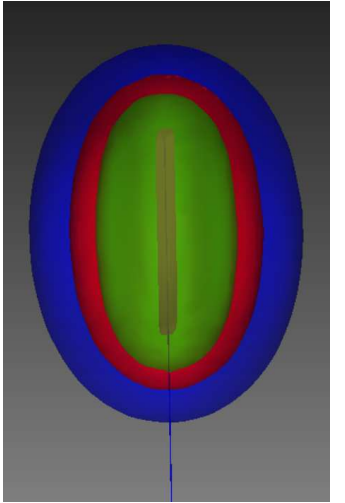

(b) Theoretical iceball shape

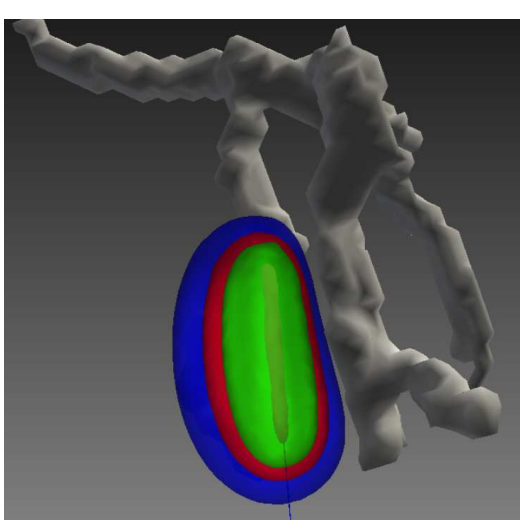

(c) Deformed iceball shape

Figure 2. Iceball deformation in the vicinity of a large vessel (gray). In green, red and blue respectively the $-40^{\circ} \mathrm{C}-20^{\circ} \mathrm{C}$ and $0^{\circ} \mathrm{C}$ isotherm surfaces

\subsection{Optimization results}

Optimization time lapse is directly related to the number of iterations for all optimizers except SM. This method builds and refines a surrogate of the domain space using interpolation methods in each stage and then optimizes the computed model. The most time consuming part of the optimization process is the resolution of the bioheat equation. For all optimizers, it is computed once per iteration. It takes 5 seconds with the spatial dimensions mentioned above on a machine using Intel core i7 3.4 with 16 GB RAM to simulate a 10 minutes cryoablation treatment. An example of an iceball produced by 3 needles around the small tumor is shown on Fig.3. On the right part of the image, we can observe that a small part of tumor (in red) is still visible outside the iceball (blue), meaning that the tumor is not entirely covered, which can be a cause of recurrence of the cancer.

In order to test the performance of each solver, we compared their speed and accuracy in different conditions: a small tumor with 3 and 5 needles, and a large tumor with 5 and 8 needles. For a fair comparison, the bottom corner of the search 

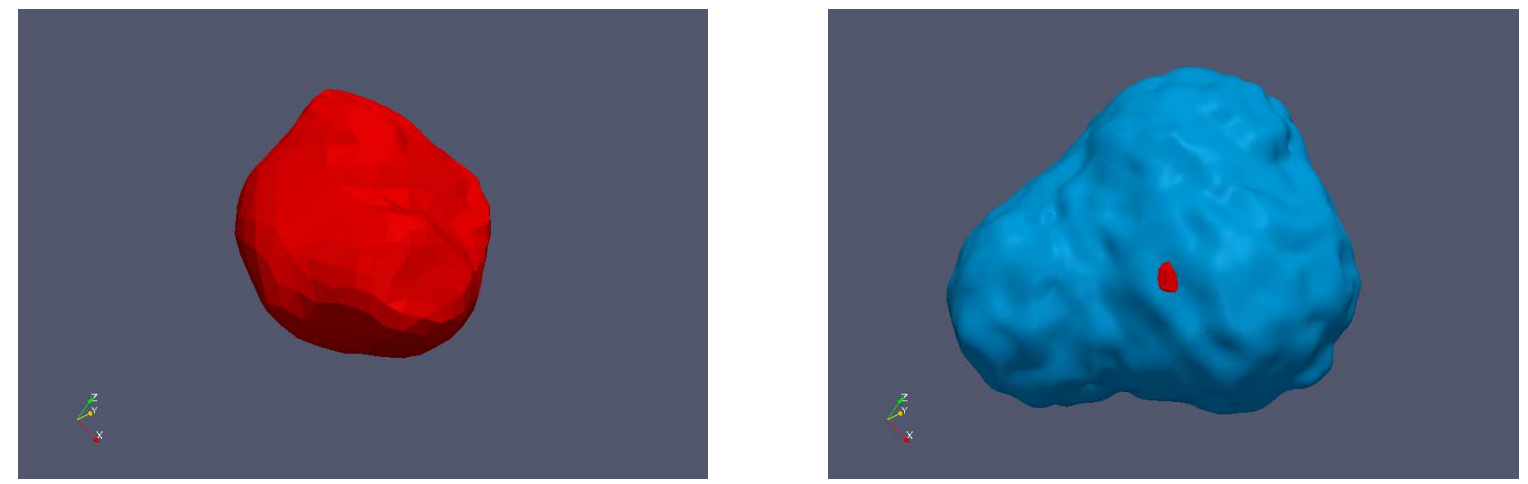

Figure 3. Example of aggregated iceball (left) after a heat propagation simulation for the small tumor (right) and 3 needles. This configuration is not completely optimal as a part of the tumor is outside the iceball.
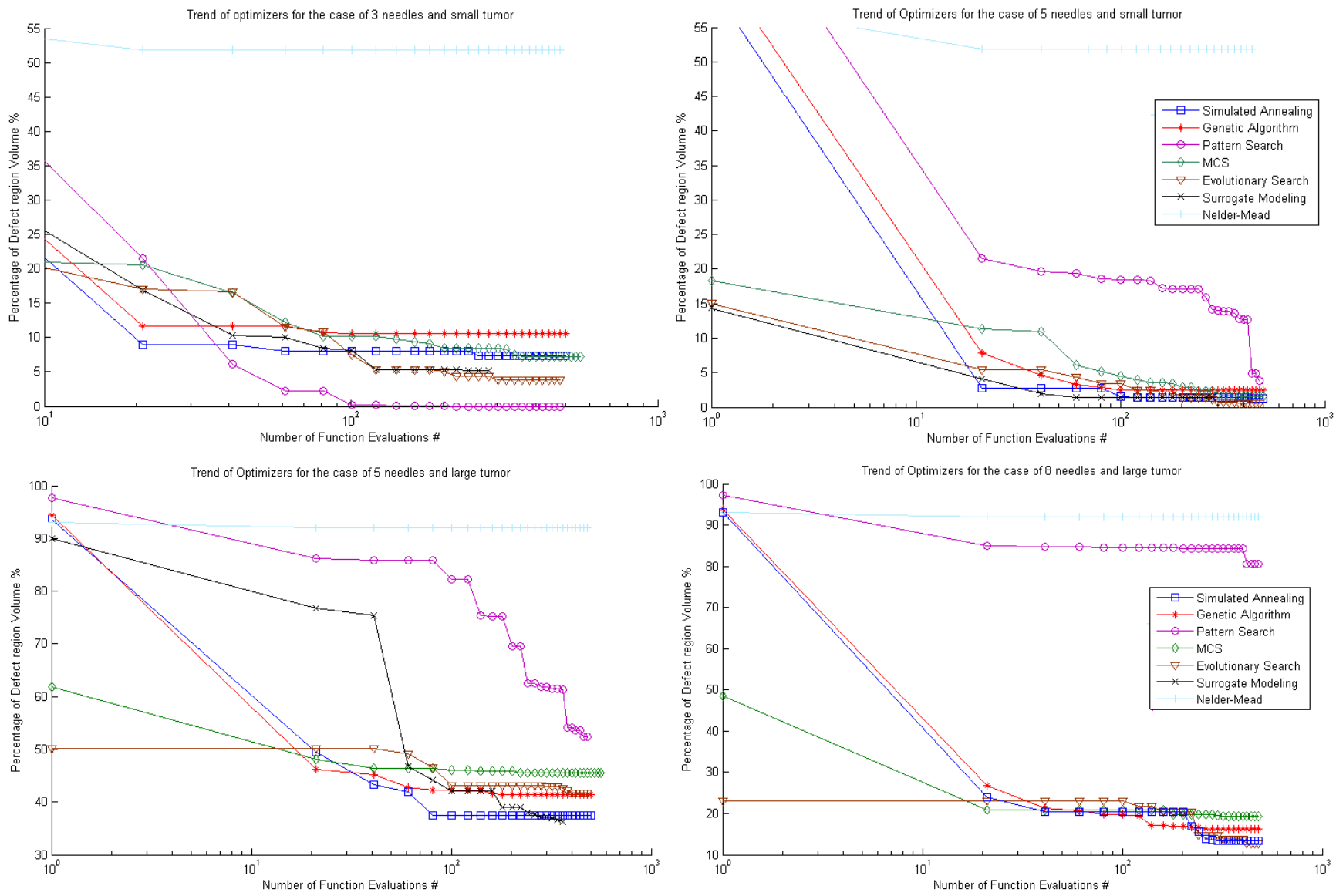

Figure 4. Trend of 7 optimizers for the case of small (top) and large (bottom) tumor, for small (left) and large (right) number of needles. Vertical axis shows the percentage of defect volume while horizontal axis shows the number of iterations, with a maximum of 500 iterations

domain was selected as an initial point for required methods like Simplex, Simulated annealing and Pattern search. Fig.4 shows the profiles of the selected solvers. These profiles sketch the percentage of defect region relatively to the number of iterations. Iterations were stopped at a maximum of 500 .

When comparing the speed of each experimental design, we were interested in finding which method had the minimum of defect volume in its first one hundred iterations. This was obtained from the profiles of optimizers cut at one hundred iterations, and shown in a more synthetic way on Fig.5(a). On this figure, speed comparison is demonstrated for the four different problem sizes mentioned above. GSS (Pattern Search) method was prior to others in the first state. Thanks to a large ratio iceball volume / tumor volume and a lower number of needles, we were facing a simple problem which could be solved easily by a local deterministic solver. NM solver was apparently stuck in a local minima due to its initial value in the border of the search field. Other solvers had more or less the same speed in this scenario leaded by SA. By increasing tumor size, deterministic methods decreased their performance especially for the GSS method, also visible for MCS, but speed of 


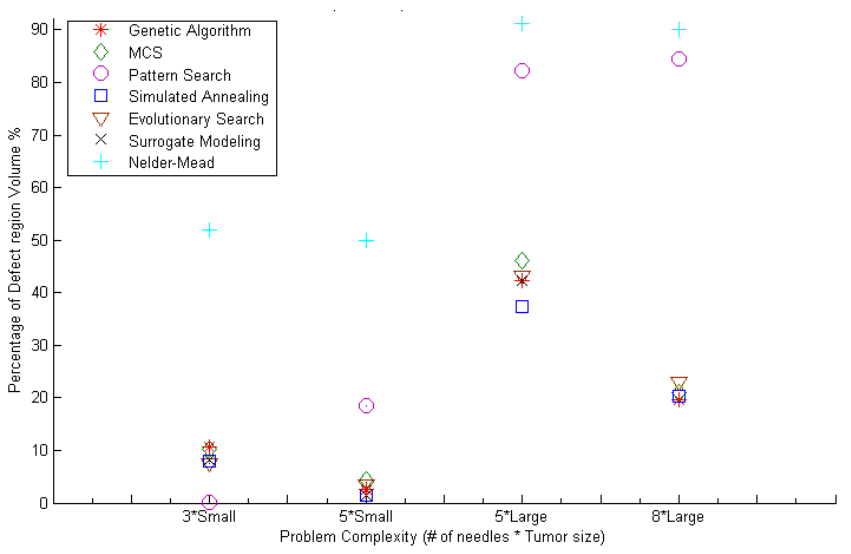

(a) Speed

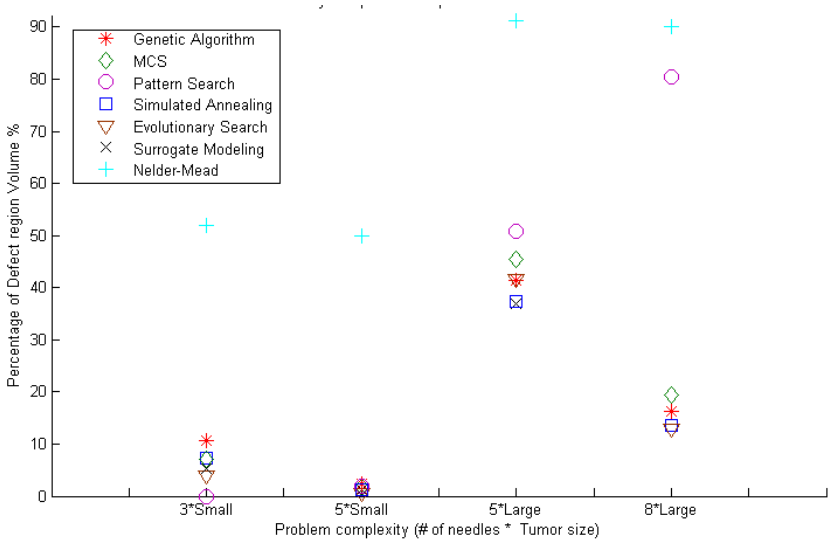

(b) Accuracy

Figure 5. Speed and accuracy comparison of 7 optimizers

optimization for other solvers was not affected by changing the search domain.

In a more general way, when studying Fig.4, the number of iterations required by all but NM to converge was proportional to problem size, which was expected due to their search logic [26]. In most cases, a convergence was found in around 100 iterations for the small tumor or 300 iterations for the large tumor. If we consider an average of 5 seconds per iteration, we obtain computation times around $8 \mathrm{mn}$ for the small tumor and $24 \mathrm{mn}$ for the large one. Our objective being to maintain the process under a reasonable time to be usable in clinical routine, we studied the precision of the optimizers when restricting the process to a maximum of 100 iterations. On Fig.5(a), we easily observe that a higher number of iceballs allows to find in a short time a better coverage of the tumor.

For the accuracy comparison we also looked for the method providing the lowest defect volume regardless of number of iterations. This can be observed on the trends of Fig.4, and is also presented in a more synthetic way on Fig.5(b). In general, evolutionary methods had a better flexibility than heuristic methods like MCS and GSS. Simulated annealing showed a rapid convergence among global methods, but it has the drawback to be dependent on its initial point. Therefore, the results were not always good with different initial points and tumor shapes. MCS had more or less good results in long iterations regardless of problem complexity due to its global design. SM had the same performance of speed and accuracy comparing to SA and better than GA. Also it was more robust when changing conditions of tumor shapes or initial point. SM had the same speed and accuracy as SA, the strength of this approach lying in the generality of its formulation since SM is independent of the physical interpretation. In other words, through SM, it is possible to set different kinds of free planning parameters without changing the optimization technique. However, its drawback is the complex inner computations, which would be annoying for a large number of input variables. A too large number of needles was fixed to 20 in the literature. However, we succeeded in using this method with 25 variables for the case of 5 needles, each having 5 degrees of freedom. For 8 needles with 5 degrees of freedom for each, we would reach 40 parameters to optimize which is too complex. This is why SM is not shown on the lower right part of Fig.4.

In order to improve computation times while preserving the accuracy, we also studied a hybrid method. A low fidelity model of iceballs was created. In this model each iceball was approximated by a simple ellipsoid with same dimensions as the simulated one in its resting state (no vicinity heat source available except the needle tip). Using this simplified model, it was possible in a first step to run optimizers with a larger number of iterations and find a better initial position for the needles with less computational cost, and then include high cost bioheat computations in a second step. Such a kind of 2-steps hybrid algorithm was tested by Tanaka [10] for the bubblepacking method. In the first step we could use any of the optimizers to deal with the low fidelity model but after some tests, Pattern search and Nelder-Mead converged to local minima and were omitted from the list. For the second step, methods accepting initial value were used: among our list, Pattern search, Nelder-Mead and Simulated annealing were selected. The most complex problem with 8 needles and large tumor was a good candidate to test the efficiency of this hybrid method. The results are shown on Table 1 for three different optimizers in the first phase and three solvers for the second phase.

In order to show the improvement of the two steps versus one step method we compared the required number of iterations to find a specific precision for each combination. We tried to compare the best result of each combination and due to the superiority of some combinations to others there is a specific precision for each combination of methods. In this table, the necessary number of iterations for the second step is shown on the left column. For each of them, the number of iterations needed to reach the mentioned precision if we had used it alone directly with high fidelity model is presented. For example if we use Genetic algorithm to optimize the low fidelity model and use Simulated annealing for second step we just need 16 iterations to reach a precision of $16.9 \%$, but the same precision using only Simulated annealing with high fidelity model needs 221 iterations. Considering 5 seconds for each simulation we have saved more than 17 minutes. For Nelder-Mead and Pattern search, as shown in Fig 4, the precision in Table 1 was not achieved in less than 500 iterations. Transition from 
Table 1. Comparison of 9 different combinations for hybrid algorithm, tested on large tumor with 8 needles

\begin{tabular}{|c|c|c|c|c|c|c|c|}
\hline \multirow{2}{*}{$\begin{array}{c}\text { Method } \\
\text { 2nd step }\end{array}$} & \multirow{2}{*}{$\begin{array}{c}\text { Nb. of } \\
\text { iterations } \\
\text { 2nd step }\end{array}$} & \multicolumn{2}{|c|}{ Method 1st step } & \multicolumn{2}{|c|}{ Simulated annealing } & \multicolumn{2}{c|}{ Genetic Algorithm } \\
\cline { 3 - 8 } & & Precision & $\begin{array}{c}\text { Nb. iter. if } \\
\text { 2nd alone }\end{array}$ & Precision & $\begin{array}{c}\text { Nb. iter. if } \\
\text { 2nd alone }\end{array}$ & Precision & $\begin{array}{c}\text { Nb. iter. if } \\
\text { 2nd alone }\end{array}$ \\
\hline Simulated Annealing & 16 & $22.4 \%$ & 23 & $16.9 \%$ & 221 & $17 \%$ & 218 \\
Nelder-Mead & 127 & $18.9 \%$ & - & $21.0 \%$ & - & $14.6 \%$ & - \\
Pattern Search & 105 & $20.4 \%$ & - & $20.3 \%$ & - & $19.8 \%$ & - \\
\hline
\end{tabular}

first step to second step had an interesting feature. For instance with Genetic algorithm method, the best percentage of defect region for low fidelity was $35 \%$, then by solving the bioheat equation with the calculated initial points, the percentage decreased to $27 \%$. This difference is due to the heat sink effect of the vessels which is not predictable and depends on the geometry and relative locations of the tumor and vessels.

Overall, with the hybrid methods, we greatly improved computation times for large tumors cases. As the 1st step optimization is nearly instantaneous (or at least has a negligible computation time), we can observe that whatever the 2nd step optimizer, the number of iterations went down to less than 130, i.e. approximately $10 \mathrm{mn}$. SA method gives the fastest results, however the difficulty of finding the most appropriate parameters for this method doesn't make it a good candidate for a use in real conditions of the medical application. GSS and NM will be considered in our future works.

\section{Conclusion and future works}

In this study, we compared seven derivative free optimization methods. The speed and accuracy of each method was investigated relatively to different numbers of needles and tumor sizes. Simulated annealing was found as the fastest for medium and large problems and in general stochastic methods were robust in different problems sizes. A hybrid method was introduced to speed up the convergence and a comparison of several possible combinations was done. In this context, SA had the fastest convergence and Nelder-Mead method improved the most by presenting a good initial point. We have demonstrated our tests by solving bioheat equation inside the optimization process for a 3D cryosurgical planning of two tumor sets of small and large size. We found that the hybrid method allows us to have an optimization in good conditions of precision in a computation time reasonable enough to be used in clinical routine.

The objective function we used was defined based on the defect volume value and did not consider its shape. We believe that taking into account the shape of the objective function will lead to higher precision and lower number of iterations in future works. In order to solve the mentioned convergence problem for large tumors in an acceptable time we are thinking about experimenting smarter routines like supervised methods or multi-stage optimization.

\section{Acknowledgments}

The authors would like to thank Region Alsace and CNRS for the financial support of this work.

\section{References}

1. Fuchs K. H.: Minimally invasive surgery. Endoscopy 34(2) (2002) 154-159

2. Rubinsky B.: Cryosurgery. Annual review of biomedical engineering 2(1) (2000) 157-187

3. Deng Z.-S. and Liu, J.: Numerical simulation of 3-D freezing and heating problems for combined cryosurgery and hyperthermia therapy. Numerical Heat Transfer, Part A: Applications 46(6) (2004) 587-611

4. Altrogge I., Kröger T., Preusser T., Büskens C., Pereira P.L., Schmidt D., Weihusen A. and Peitgen H.-O. : Towards optimization of probe placement for radio-frequency ablation. Medical Image Computing and Computer-Assisted InterventionMICCAI (2006) 486-493

5. Baegert C., Villard C., Schreck P., Soler L. and Gangi A.: Trajectory optimization for the planning of percutaneous radiofrequency ablation of hepatic tumors. Computer Aided Surgery 12(2) (2007) 82-90

6. Villard C., Soler L. and Gangi A.: Radiofrequency ablation of hepatic tumors: simulation, planning, and contribution of virtual reality and haptics. Computer Methods in Biomechanics and Biomedical Engineering 8(4) (2005) 215-227

7. Chen C.C.R., Miga M.I. and Galloway R.L.: Optimizing electrode placement using finite-element models in radiofrequency ablation treatment planning. Biomedical Engineering, IEEE Transactions on 56(2) (2009) 237-245

8. Keanini R. and Rubinsky B.: Optimization of multiprobe cryosurgery. Heat Transfer 114(4) (1992) 796-801

9. Baissalov R., Sandison G., Reynolds D. and Muldrew K.: Simultaneous optimization of cryoprobe placement and thermal protocol for cryosurgery. Physics in medicine and biology 46(7) (2001) 1799

10. Tanaka D., Shimada K. and Rabin Y.: Two-phase computerized planning of cryosurgery using bubble-packing and force-field analogy. Journal of biomechanical engineering 128(1) (2006) 49-58 
11. Tanaka D., Shimada K., Rossi M.R. and Rabin Y.: Cryosurgery planning using bubble packing in 3d. Computer methods in biomechanics and biomedical engineering 11(2) (2008) 113-121

12. Giorgi G., Avalle L., Brignone M., Piana M. and Caviglia G.: An optimisation approach to multiprobe cryosurgery planning. Computer Methods in Biomechanics and Biomedical Engineering (2011) 1-11

13. Zhang Y.T., Liu J. and Zhou Y.X.: Pilot study on cryogenic heat transfer in biological tissues embedded with large blood vessels. Forschung im Ingenieurwesen 67(5) (2002) 188-197

14. Pennes H.H.: Analysis of tissue and arterial blood temperatures in the resting human forearm. Journal of applied physiology $\mathbf{1}(2)$ (1948) 93-122

15. Vanderplaats G.N.: Numerical optimization techniques for engineering design: with applications. Volume 32. McGraw-Hill New York (1984)

16. Rios, L.M., Sahinidis, N.V.: Derivative-free optimization: A review of algorithms and comparison of software implementations. Journal of Global Optimization (2012) 1-47

17. Nelder J.A. and Mead R.: A simplex method for function minimization. The computer journal 7(4) (1965) 308-313

18. Kolda T.G., Lewis R.M. and Torczon V.: Optimization by direct search: New perspectives on some classical and modern methods. SIAM review 45(3) (2003) 385-482

19. Huyer W. and Neumaier A.: Global optimization by multilevel coordinate search. Journal of Global Optimization 14(4) (1999) $331-355$

20. Barton R.R.: Metamodeling: a state of the art review. Proceedings of the 26th conference on Winter simulation (1994) 237-244

21. Powell M.J.: Recent research at Cambridge on radial basis functions. Springer (1999)

22. Sacks J., Welch W.J., Mitchell T.J. and Wynn H.P.: Design and analysis of computer experiments. Statistical science 4(4) (1989) 409-423

23. Powell M.J.: Uobyqa: unconstrained optimization by quadratic approximation. Mathematical Programming 92(3) (2002) 555-582

24. Holland J.H.: Adaptation in natural and artificial systems: An introductory analysis with applications to biology, control, and artificial intelligence. U Michigan Press (1975)

25. Hansen N.: The CMA evolution strategy: a comparing review. Towards a new evolutionary computation 192 (2006) 75-102

26. Lacksonen, T.: Empirical comparison of search algorithms for discrete event simulation. Computers \& Industrial Engineering 40(1) (1975) 133-148 